\title{
Составной генераторный термоэлемент на рабочий диапазон температур $30-320^{\circ} \mathrm{C}$
}

\author{
(C) А.И. Сорокин \\ АО „Гиредмет“, \\ 119017 Москва, Россия \\ E-mail: almaz_gx@mail.ru
}

(Получена 27 декабря 2016 г. Принята к печати 12 января 2017 г.)

Исследована возможность повышения кпд термоэлементов в диапазоне температур $30-320^{\circ} \mathrm{C}$, используя для этого подход, связанный с созданием составных ветвей термоэлемента. Составные ветви $n$ - и $p$-типа были построены из низкотемпературных термоэлектрических материалов на основе теллурида висмута и добавки среднетемпературного материала на основе PbTe и GeTe соответственно. Формирование составных ветвей осуществляли методом искрового плазменного спекания (ИПС). Этот метод обеспечивает контактное сопротивление на уровне $\leq 10$ мкОм - см. Проведено сравнение свойств составного термоэлемента с традиционным. Кпд составных термоэлементов по сравнению с традиционным увеличился практически на $70 \%$ и составил $5.3 \%$ в рабочем диапазоне $30-320^{\circ} \mathrm{C}$.

DOI: $10.21883 /$ FTP.2017.07.44633.19

Одним из основных способов получения электроэнергии является создание альтернативных, прежде всего возобновляемых источников энергии. Важное место в решении этой проблемы принадлежит преобразованию тепла в электроэнергию с использованием ТЭГ. Интерес к ТЭГ объясним благодаря преимуществам, которыми он обладает, таким как, прямое преобразование тепла в электроэнергию, бесшумность, долгий срок службы и т.д.

К числу недостатков термоэлектрического генератора следует отнести его низкую эффективность (кпд - $\eta$ ). Максимальная эффективность устройства может быть записана в виде

$$
\text { кпдтэ }=\frac{T_{\Gamma}-T_{\mathrm{X}}}{T_{\Gamma}}\left[\frac{\sqrt{1+(Z T)_{\text {средн. }}}-1}{\sqrt{1+(Z T)_{\text {средн. }}}+T_{\mathrm{X}} / T_{\Gamma}}\right],
$$

где $T_{\Gamma}$ - температура горячей стороны, $T_{\mathrm{X}}$ - температура холодной стороны, $(Z T)_{\text {средн. }}-$ среднее значение безразмерной термоэлектрической эффективности для рабочего температурного диапазона (от $T_{\mathrm{X}}$ до $T_{\Gamma}$ ).

Относительно низкий кпд термоэлектрического генератора сдерживает широкое использование генераторных модулей в современной технике. Повышение эффективности термоэлектрического преобразования энергии является ключевой проблемой в термоэлектрическом материаловедении. Если посмотреть на формулу (1), то из нее следует, что для повышения кпд необходимо, с одной стороны, расширить рабочий диапазон ТЭГ, а с другой - увеличить $Z T$. Характерной особенностью температурной зависимости $Z T$ является то, что эта величина имеет максимум в рабочей области температур. Положение этого максимума зависит от концентрации носителей заряда. Поэтому для получения наилучших результатов требуется изменять концентрацию носителей заряда вдоль ветви таким образом, чтобы каждая из ее частей имела бы оптимальные свойства.
Эффективность термоэлектрических материалов достигает максимальных величин в довольно ограниченной температурной области, поэтому они обычно подразделяются по температурной области их применения на низкотемпературные $\left(T_{\mathrm{X}}<300^{\circ} \mathrm{C}\right)$, среднетемпературные $\left(300<T_{\Gamma}<600^{\circ} \mathrm{C}\right)$ и высокотемпературные $\left(T_{\Gamma}>600^{\circ} \mathrm{C}\right)$. Так как единого термоэлектрического материала, эффективного во всех температурных диапазонах, не существует, то при создании термоэлектрических генераторов, работающих в широком диапазоне температур, термоэлектрическая ветвь должна состоять из сегментов различных материалов. Эти сегменты в свою очередь могут состоять из секций с различной концентрацией носителей заряда, чтобы обеспечить их работу в оптимальных условиях $[1,2]$.

В работе изучается составной термоэлемент, состоящий из $\mathrm{Bi}_{2} \mathrm{Te}_{3}(n 1) / \mathrm{Bi}_{2} \mathrm{Te}_{3}(n 2) / \mathrm{PbTe} n$-ветви и $\mathrm{Bi}_{2} \mathrm{Te}_{3}(p 1) / \mathrm{Bi}_{2} \mathrm{Te}_{3}(p 2) / \mathrm{GeTe} p$-ветви для наиболее востребованного на данный момент рабочего температурного диапазона $30-320^{\circ} \mathrm{C}$.

Исходные секции низкотемпературного термоэлектрического материала на основе $\mathrm{Bi}_{2} \mathrm{Te}_{3} n$ - и $p$-типа проводимости изготавливали методом горячей экструзии [3]. Среднетемпературные секции $\mathrm{PbTe}$ и GeTe - методом искрового плазменного спекания. На рис. 1 показаны температурные зависимости $Z T$ для двух ветвей.

Формирование составных ветвей $n$ - и $p$-типа осуществляли на установке ИПС (SPS511S, Япония). При кажущейся простоте сопряжение секций различного состава представляет технически сложную задачу. Поэтому необходимо было решить ряд важных задач: вопервых, нужно соединить секции между собой, обеспечив минимальное контактное сопротивление; во-вторых, соединения между секциями должны обладать высокими адгезивными свойствами.

Готовые секции в порядке повышения концентрации носителей укладывали в графитовую пресс- 

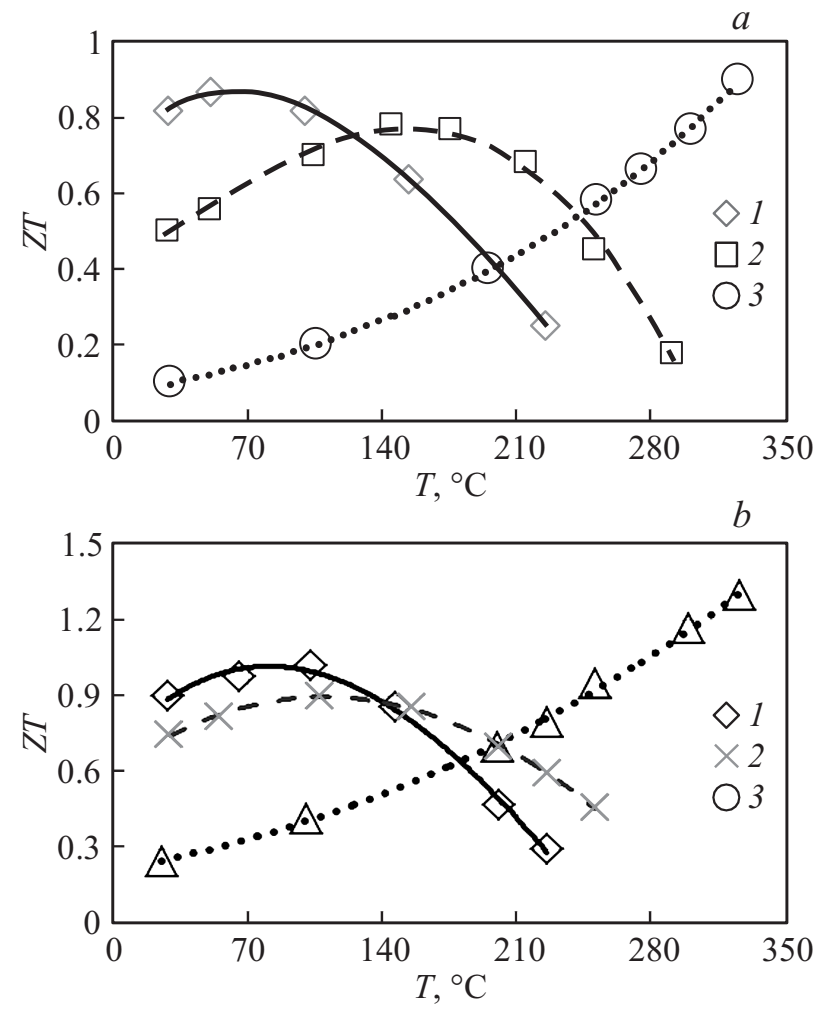

Рис. 1. Температурные зависимости $Z T$ для ветвей $n$ - $(a)$ и -типа $(b) .1-\mathrm{Bi}_{2} \mathrm{Te}_{3}(n 1 / p 1), 2-\mathrm{Bi}_{2} \mathrm{Te}_{3}(n 2 / p 2), 3-$ $\mathrm{PbTe} / \mathrm{GeTe}$.

форму диаметром 20 мм. В качестве антидиффузионнойсоединительной прослойки между секциями $\mathrm{Bi}_{2} \mathrm{Te}_{3}$ и $\mathrm{PbTe} / \mathrm{GeTe}$ использовали специально подобранную нанокомпозитную смесь на основе металл + связующее $\mathrm{CoSn}$ „NCS32“. Данная смесь позволила обеспечить надежное соединение секций разнородных материалов с высокими прочностными характеристиками и электрофизическими свойствами. Соединение же секций на основе $\mathrm{Bi}_{2} \mathrm{Te}_{3}$ осуществляли напрямую, при этом необходимо было учесть высокую текучесть термоэлектрического материала при повышенных температурах [4].

Важным технологическим параметром в процессе формирования составных ветвей является температура, так как она определяет пластическое течение материалов и скорость диффузионного обмена на границе. Поэтому формирование составных ветвей осуществляли

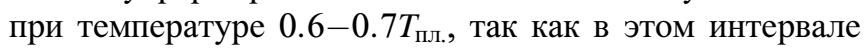
температур велика интенсивность диффузионных процессов. На границе раздела диффузионного соединения $\mathrm{Bi}_{2} \mathrm{Te}_{3}(n 1, p 1) / \mathrm{Bi}_{2} \mathrm{Te}_{3}(n 2, p 2)$ непровар не наблюдался.

Преимущество данного способа формирования составной ветви перед способом, основанным на использовании припоя, состоит в том, что в системе „основной материал-промежуточный (антидиффузионный) слой-основной материал“ нет явной границы раздела и тем самым резкого изменения физико-химических и механических свойств.
Для получения высоких характеристик термоэлемента критичным является совместимость материалов секций по теплофизическим и электрическим свойствам. Условия теплового баланса составной ветви требуют, чтобы тепловой поток, выходящий из одной секции, был равен тепловому потоку, входящему в последующую секцию. Для достижения максимального кпд оба этих тепловых потока должны соответствовать режиму максимального кпд в секциях. Кроме того, токи в каждой из секций должны соответствовать режиму максимального кпд и совпадать. Для согласования этих двух рабочих параметров есть только одна переменная - длина секции. На рис. $2, a, b$ показана схема формирования экспериментальной ветви $p$-типа. На рис. $2, c-$ фото ветвей.

Оптимизация составной ветви сводится к подбору длин ее отдельных секций. Эти длины устанавливаются таким образом, чтобы каждая секция обеспечивала бы максимально высокий кпд.

Важным требованием к составным ветвям являются низкое контактное электрическое сопротивление и высокая адгезия слоев друг к другу. Для контроля этих параметров из готовых пластин вырезали ветви сечением $4 \times 4$ мм.

Для оценки величины адгезии многослойных ветвей $n$ и $p$-типа проводимости, полученных в едином процессе искрового плазменного спекания, проводились испытания на разрыв. Для этого к торцам ветвей приклеиванием прочно присоединялись „металлические башмаки“, с помощью которых определялось разрушающее усилие. Испытание проводили на стенде PCE-FTS 50 (PCE, Германия). Проведенные испытания показали, что разрыв
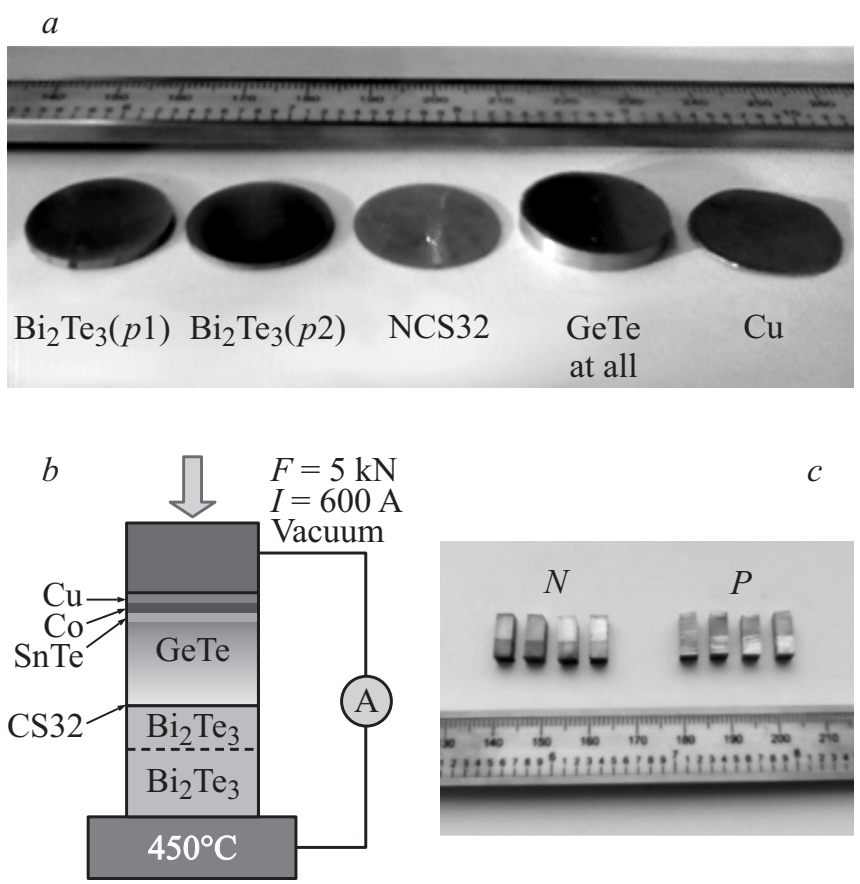

Рис. 2. $a-$ изображения секций, $b-$ схема укладки экспериментальной ветви $p$-типа, $c-$ составные ветви. 


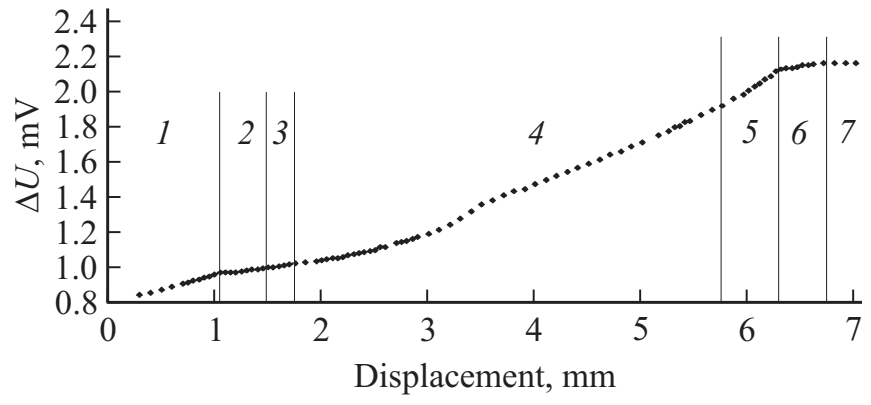

Рис. 3. Изменение потенциала вдоль участков составной ветви p-типа: $1-\mathrm{Bi}_{2} \mathrm{Te}_{3}(p 1), 2-\mathrm{Bi}_{2} \mathrm{Te}_{3}(p 2), 3-\mathrm{NCS} 32,4-$ GeTe, $5-\mathrm{SnTe}, 6-\mathrm{Co}, 7-\mathrm{Cu}$.

происходит по наименее прочному материалу, в нашем случае это PbTe и GeTe. C учетом погрешности измерения прочности на разрыв можно считать, что величина этого параметра для ветвей $n$ - и $p$-типа различается незначительно и составляет $\geq 100$ кг/ $\mathrm{cm}^{2}$.

Контактное сопротивление составной ветви контролировали по измерению скачка потенциала в области контакта при комнатной температуре. На рис. 3 представлена зависимость изменения потенциала по длине p-ветви.

Скачок потенциала $\Delta U$ на контактном сопротивлении связан с пропускаемым через образец током соотношением

$$
\Delta U=j \rho_{\text {cont }},
$$

где $j-$ плотность тока через образец, $\rho_{\text {cont }}-$ контактное сопротивление.

Расчет контактного сопротивления на границе двух секций $\mathrm{Bi}_{2} \mathrm{Te}_{3} / \mathrm{Bi}_{2} \mathrm{Te}_{3}$ показал, что величина его составляет $\sim 8$ мкОм · см. Близкие величины мы наблюдаем и на переходах $\mathrm{Bi}_{2} \mathrm{Te}_{3} / \mathrm{NCS} 32 / \mathrm{GeTe} / \mathrm{SnTe}$. Таким образом, подобранная нами антидиффузионная прослойка NCS32 обеспечивает надежный низкоомный контакт двух разнородных материалов. Контактное сопротивление на переходе $\mathrm{SnTe} /$ Со составляет $\sim 22$ мкОм - см. В составной ветви $n$-типа контактное сопротивление меняется от $\sim 8$ до 20 мкОм · см.

Суммируя результаты по адгезии и контактному сопротивлению как между „секция-секция“, так и между „секция/антидиффузионный слой/секция“, можно заключить, что составные ветви, скоммутированные искровым плазменным спеканием, обладают высокой адгезией слоев на разрыв и, как следствие, низким контактным сопротивлением. Это позволит минимизировать потери тепла и тока на границах, что в свою очередь, скажется на увеличении эффективности этих ветвей и термоэлемента соответственно.

Серия термоэлементов с составными ветвями, состоящими из одной, двух и трех секций, тестировались в максимальном перепаде температур. Наивысший кпд 5.3\% показали трехсекционные ветви. Рост по сравнению с традиционными ветвями составляет примерно 70\%, в первую очередь за счет лучшей оптимизации как самой ветви, так и материалов секций.

Работа выполнена при поддержке Министерства образования и науки РФ (субсидия № RFMEFI57914X003914.579.21.0039).

\section{Список литературы}

[1] Zhongliang Ouyang, Dawen Li. Sci. Rep., 6, 24123 (2016).

[2] Hee Seok Kim, Keiko Kikuchi, Takashi Itoh, Tsutomu Lida, Minoru Taya. Mater. Sci. Engin. B, 185, 45 (2014).

[3] М.Г. Лаврентьев, М.В. Меженный, В.Б. Освенский, А.И. Простомолотов. МЭТ, 3, 35 (2012).

[4] M.G. Lavrentev, V.B. Osvenskii, Yu.N. Parkhomenko, G.I. Pivovarov, A.I. Sorokin, L.P. Bulat, H.-S. Kim, I.T. Witting, G.J. Snyder, V.T. Bublik, N.Yu. Tabachkova. APL Materials, 9 (2016).

Редактор Г.А. Оганесян

\section{Segmented thermoelectric unicouple at operating temperature range $30-320^{\circ} \mathrm{C}$}

\section{A.I. Sorokin}

\section{"Giredmet" JSC, 119017 Moscow, Russia}

Abstract The purpose of the work is to study the possibility to increase the efficiency of the thermocouples in the temperature range of $30-320^{\circ} \mathrm{C}$, using approach associated with the creation of segmented thermoelectric unicouple. The thermoelectric unicouples of $n$ - and $p$-types were constructed from low-temperature thermoelectric materials based on bismuth telluride and additional medium-temperature thermoelectric materials $\mathrm{PbTe}$ and $\mathrm{GeTe}$ respectively. The thermoelectric unicouple was fabricated by spark plasma sintering (SPS). The method of SPS allowed to keep a contact resistance at the level of $\leq 10 \mu \Omega \mathrm{cm}$. The comparison of the properties of segmented and traditional unicouple was made. The efficiency of segmented unicouple was about 1.4 times greater, compared to the value of traditional one, and reached $5.5 \%$ in the operating temperature range of $30-320^{\circ} \mathrm{C}$. 